OPEN

SUBJECT AREAS:

PLANT MOLECULAR

BIOLOGY

PLANT GENETICS

CHLOROPLASTS

LIGHT RESPONSES

Received

1 September 2014

Accepted

12 March 2015

Published

15 April 2015

Correspondence and requests for materials should be addressed to

H.G. Igaohongbo@ bjfu.edu.cn)

* These authors contributed equally to this work.

\section{Arabidopsis FHY3/CPD45 regulates far-red light signaling and chloroplast division in parallel}

\author{
Ning Chang*, Yuefang Gao*, Lin Zhao, Xiaomin Liu \& Hongbo Gao
}

College of Biological Sciences and Biotechnology, Beijing Forestry University, Beijing, 100083, China.

CPD45 (chloroplast division45), which is also known as FHY3 (far-red elongated hypocotyl3), is a key factor in the far-red light signaling pathway in Arabidopsis. We previously showed that FHY3/CPD45 also regulates chloroplast division. Because light is also a regulator of chloroplast development and division, we sought to clarify the relationship between far-red light signaling and chloroplast division pathways. We found that the chloroplast division mutant arc5-3 had no defect in far-red light sensing, and that constitutive overexpression of $A R C 5$ rescued the chloroplast division defect, but not the defect in far-red light signaling, of cpd45. fhyl, which is defective in far-red light signaling, exhibited normal chloroplast division.

Constitutive overexpression of FHY1 rescued the far-red light signaling defect, but not the chloroplast division defect, of cpd45. Moreover, ARC5 and FHY1 expression were not affected in fhyl and arc5-3, respectively. Based on these results, we propose that FHY3/CPD45 regulates far-red light signaling and chloroplast division in parallel by activating the expression of FHY1 and ARC5 independently. This work demonstrates how relationships between different pathways in a gene regulatory network can be explored.

$\mathrm{P}$ lants adapt their growth according to changing environmental conditions, and one of the most important environmental inputs is light. Light not only provides energy for plants via photosynthesis, but also functions as a signal that regulates plant growth and development. Five types of light receptors have been reported in plants, namely phytochromes $(\mathrm{phy})^{1}$, cryptochromes $^{2}$, phototropins ${ }^{3}$, Zeitlupe ${ }^{4}$ and UVR $8^{5,6}$. Five phys species exist in Arabidopsis thaliana, phyA to $\mathrm{E}^{1,7}$. PhyA, a red light $(620 \sim 700 \mathrm{~nm})$ and far-red light $(700 \sim$ $800 \mathrm{~nm})^{8}$ sensor, is imported into the nucleus upon light illumination, with the help of FHY1 (Far-red elongated Hypocotyl 1) (-11 $^{9}$ and its homolog FHL (FHY1-like) ${ }^{12}$. FAR1 (far-red-impaired response) ${ }^{13}$ and FHY3 (also known as CPD45) are transcription factors in the same family that are involved in far-red light signaling through activating the expression of $F H Y 1$ and $F H L^{14}$. Due to their defects in far-red light signaling, fhy 3 , fhyl, and phyA plants have elongated hypocotyls under far-red light ${ }^{15-17}$.

Chloroplasts, which evolved from endosymbiotic cyanobacteria, are not only the major site of photosynthesis, but also the major site for numerous important biosynthetic pathways, such as fatty acid biosynthesis ${ }^{18}$, amino acid biosynthesis ${ }^{19}$, and the immune response in plants ${ }^{20}$. Chloroplasts are generated through the binary fission of preexisting chloroplasts ${ }^{21}$. In mutants with chloroplast division defects, chloroplasts are generally enlarged in size and reduced in number. The large chloroplasts are readily injured by high light intensities, due to their low mobility, which also affects photosynthesis and other metabolic pathways ${ }^{22,23}$.

Many genes involved in chloroplast division have been identified. For instance, $A R C 5$, which is a nuclear gene, encodes a dynamin-related protein that is part of the chloroplast division machinery ${ }^{24}$. The arc5 mutant has enlarged and dumbbell-shaped chloroplasts. Subsequently, mutants with a similar phenotype, $c p d 45$ and $c p d 25$, were identified ${ }^{25}$. Map-based cloning revealed that CPD45 was the same gene as FHY3 and CPD25 was the same gene as FRS4, a homolog of CPD45/FHY ${ }^{25}$. Further analysis suggested that CPD45 worked cooperatively with CPD25 to activate the expression ARC5. Unlike $c p d 45$, the hypocotyl length of $c p d 25$ was similar to that of the wild type under far-red light, suggesting that CPD25 is not involved in far-red light signaling ${ }^{25}$.

Light affects many aspects of chloroplast development and function, including biogenesis, morphogenesis, movement, and biochemical metabolism ${ }^{26}$. Studies conducted in our lab and others suggest that FHY3/CPD45 not only promotes chloroplast division by activating the expression of $A R C 5$, but is also involved in light sensing by promoting the nuclear import of phyA, which is essential for its function in light signaling ${ }^{16,25,27,28}$. However, it is unclear whether FHY3/CPD45 promotes chloroplast division through FHY1 and PHYA. We addressed this question using a set of genetic experiments with related mutants and transgenic plants. Our analysis suggests that 
FHY3/CPD45 regulates chloroplast division and far-red light signaling by activating the expression of $A R C 5$ and $F H Y 1$ in parallel.

\section{Results}

Constitutive overexpression of FHY1 rescued the far-red light sensing defect, but not the chloroplast division defect, of $c p d 45$. To establish whether the effect of FHY3/CPD45 on chloroplast division is mediated by $F H Y 1$ and the far-red light signaling pathway, we constitutively overexpressed $35 S-F H Y 1$ in the cpd45 mutant background (35S-FHY1;cpd45). After incubating seeds of various genetic backgrounds for four days under far-red light, we measured the hypocotyl lengths of the young seedlings. The hypocotyls of $35 S-F H Y 1 ; c p d 45$ transgenic plants $(2.625 \mathrm{~mm})$ were significantly shorter than those of the cpd45 mutant $(13.336 \mathrm{~mm})$, but very close to those of the wild type $(2.772 \mathrm{~mm}$; Figures $1 \mathrm{a}, \mathrm{b}, \mathrm{c}$ and $2 \mathrm{a})$. However, as observed in the $c p d 45$ mutant, the chloroplasts of 40-day-old 35S-FHY1; cpd45 plants grown under white light were larger in size and reduced in number relative to the wild type (Figure $3 \mathrm{a}, \mathrm{b}$ and $\mathrm{c}$ ). We quantified the severity of the chloroplast phenotype using a best-fit linear plot of chloroplasts per cell versus cell area (Figure 4). The 35S-FHY1;cpd45 plants have a slope of 0.0048 , which is similar to that of $c p d 45(0.0059)$ and very different from that of the wild type (0.0127; Figure 4$)$. These results suggest that the constitutive overexpression of FHY1 can rescue the far-red light sensing defect of the $c p d 45$, but not the chloroplast division defect.

Interestingly, seedlings of 35S-FHY1;cpd45 were stronger than the others both in far-red light or the dark (Figure 1 and S1). Their cotyledon size was approximately twice as large as other plants. We measured the cotyledon size of the seedlings of various genetic backgrounds grown in the dark or far-red light. However, the response to far-red light of the seedlings of 35S-FHY1;cpd45 was similar to that of other plants and the cotyledon size was induced more than two times by far-red light (Figure S1).

Constitutive overexpression of $A R C 5$ rescues the chloroplast division defect, but not the far-red light signaling defect, of cpd45. To investigate whether the far-red light signaling defect of cpd45 is caused by the chloroplast division defect, we transformed $35 S$-GFP-ARC5 into the $c p d 45$ mutant. After 40 days of growth under white light, we observed the chloroplast division phenotype of leaf mesophyll cells using a microscope. We found that constitutive overexpression of GFP-ARC5 rescued the chloroplast defects of cpd45 (Figure 3d), because the chloroplast phenotype of GFP$A R C 5 ; c p d 45$ transgenic plants was very similar to that of the wild type. In our best-fit linear plot of chloroplasts per cell against cell

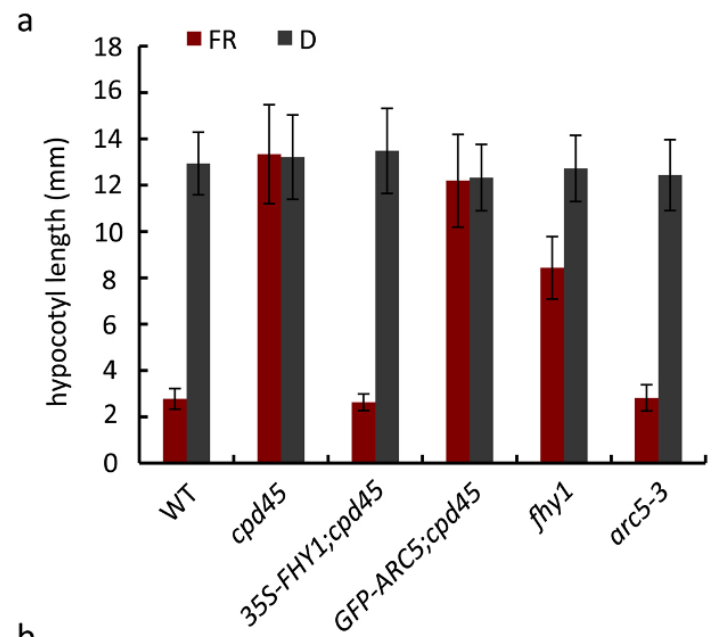

b

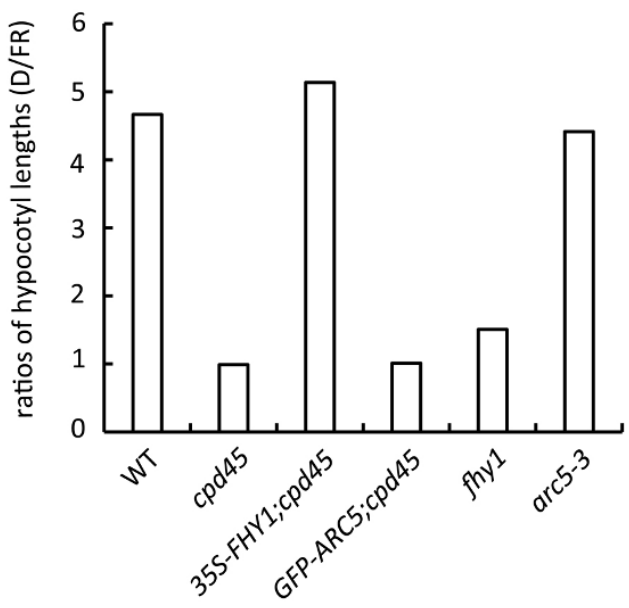

Figure $2 \mid$ Analysis of hypocotyl length. (a) Hypocotyl length of seedlings of various genetic backgrounds. FR, far-red light $\left(10 \mu \mathrm{mol} \cdot \mathrm{m}^{-2} \cdot \mathrm{s}^{-1}\right)$; $\mathrm{D}$, dark. $\mathrm{n}=30$ seedlings. Error bars represent standard deviation. Seeds were plated and hypocotyl lengths were measured after 4 days of incubation under continuous far-red light or in the dark. (b) Corresponding ratios of the hypocotyl length shown in (a).

area, the slope of GFP-ARC5;cpd45 (0.0115) was higher than that of cpd45 (0.0059), and close to that of the wild type (0.0127; Figure 4). In contrast, the hypocotyls of GFP-ARC5;cpd45 transgenic plants grown under continuous far-red light was $12.190 \mathrm{~mm}$ long, more
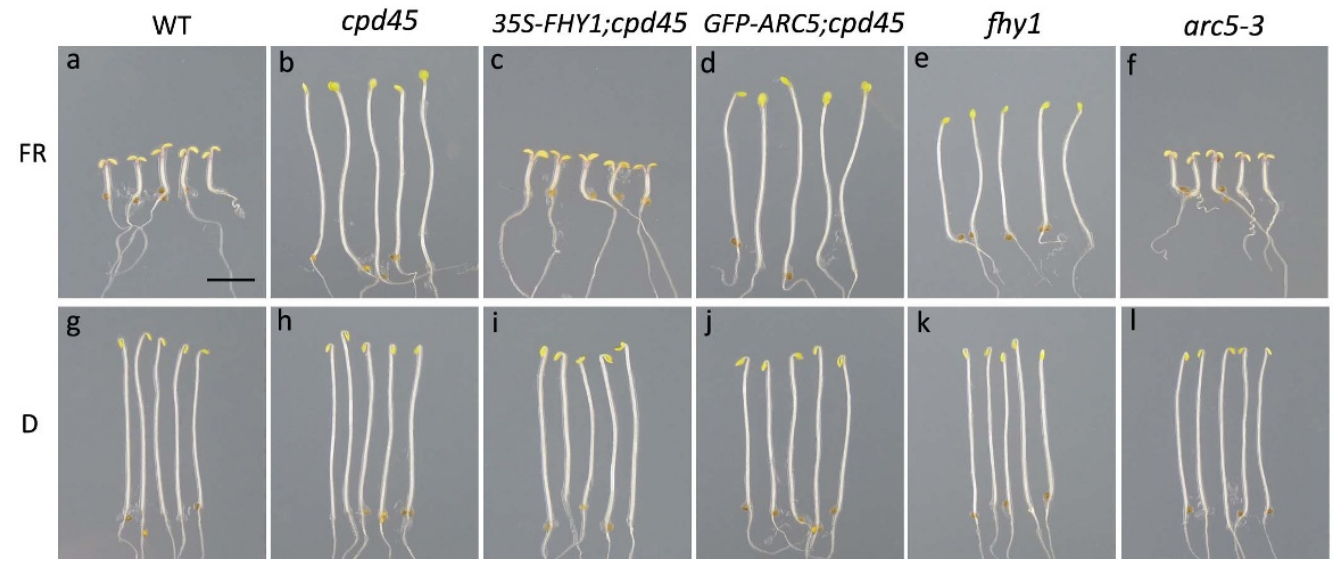

Figure 1 Seedlings of various genetic backgrounds grown in far-red light or the dark for 4 days. (a, g) Wild type. (b, h) $c p d 45$ mutant. (c, i) $c p d 45$ mutant transformed with the 35S-FHY1 transgene. ( $\mathrm{d}, \mathrm{j})$ cpd45 mutant transformed with the 35S-GFP-ARC5 transgene. (e, k) fhyl mutant.

(f, l) $\operatorname{arc5-3}$ mutant. (a-f) seedlings grown in $10 \mu \mathrm{mol} \cdot \mathrm{m}^{-2} \cdot \mathrm{s}^{-1}$ far-red light, (g-l) seedings grown in the dark. WT, wild type. Scale bar represents $3 \mathrm{~mm}$. 
WT

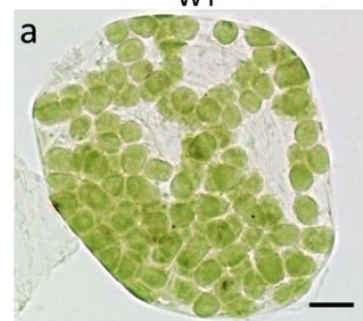

GFP-ARC5;cpd45

d

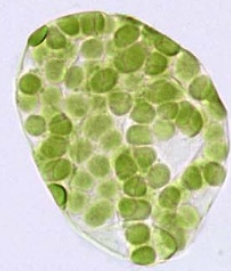

cpd45

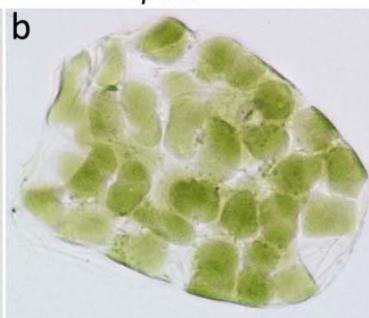

fhy1

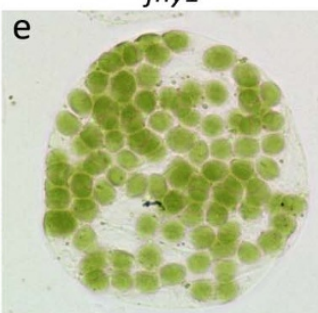

35S-FHY1;cpd45

C

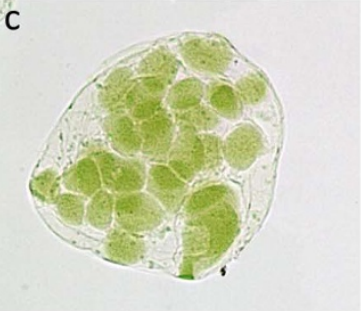

$\operatorname{arc5-3}$

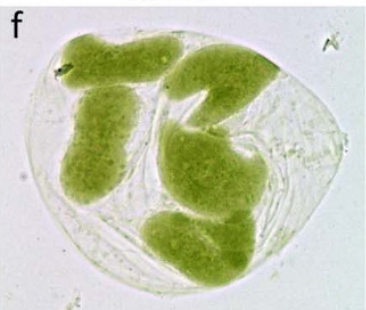

Figure 3 Chloroplast division phenotypes of mesophyll cells with different genetic backgrounds. (a) Wild type. (b) $c p d 45$ mutant. (c) $c p d 45$ mutant harboring the 35S-FHY1 transgene. (d) cpd45 mutant harboring the 35S-GFP-ARC5 transgene. (e) fhyl mutant. (f) arc5-3 mutant. Leaf tissue was sampled from 40-day-old plants. Scale bar represents $10 \mu \mathrm{m}$.

than four times as long as those of the wild type $(2.772 \mathrm{~mm})$ and close in length to those of $c p d 45$ (13.336 mm; Figures 1a, b, d and 2a). Thus, 35S-GFP-ARC5 rescues the chloroplast division defect of $c p d 45$, but not the far-red light signaling defect.

We next performed an immunoblot analysis to determine the expression level of the constitutively overexpressed GFP-ARC5 in the GFP-ARC5;cpd45 transgenic line and to compare this value with that of ARC5 in the wild type (Figure 5). We detected a band of around $100 \mathrm{kD}$ in size in the wild type. This band was absent in arc5-3 and very faint in $c p d 45$, suggesting that it corresponded with ARC5. GFP-ARC5 yielded a band of $130 \mathrm{kD}$, as expected. Based on our rough estimation, the expression level of GFP-ARC5 was at least five times greater than that of ARC5 in the wild type.

Mutations in FHY1 and ARC5 do not affect the expression of each other. We next performed semi-quantitative RT-PCR experiments to further characterize the relationship between FHY1 and ARC5 (Figure 6). To avoid saturation of the PCR amplification and for better comparison of the expression levels of the genes, the cDNA used in the experiment was serially diluted three times with a dilution ratio of 4. HTA9, which is a constitutively expressed gene, was used as a control. The expression level of FHY1 in $\operatorname{arc5}-3$ was similar to that

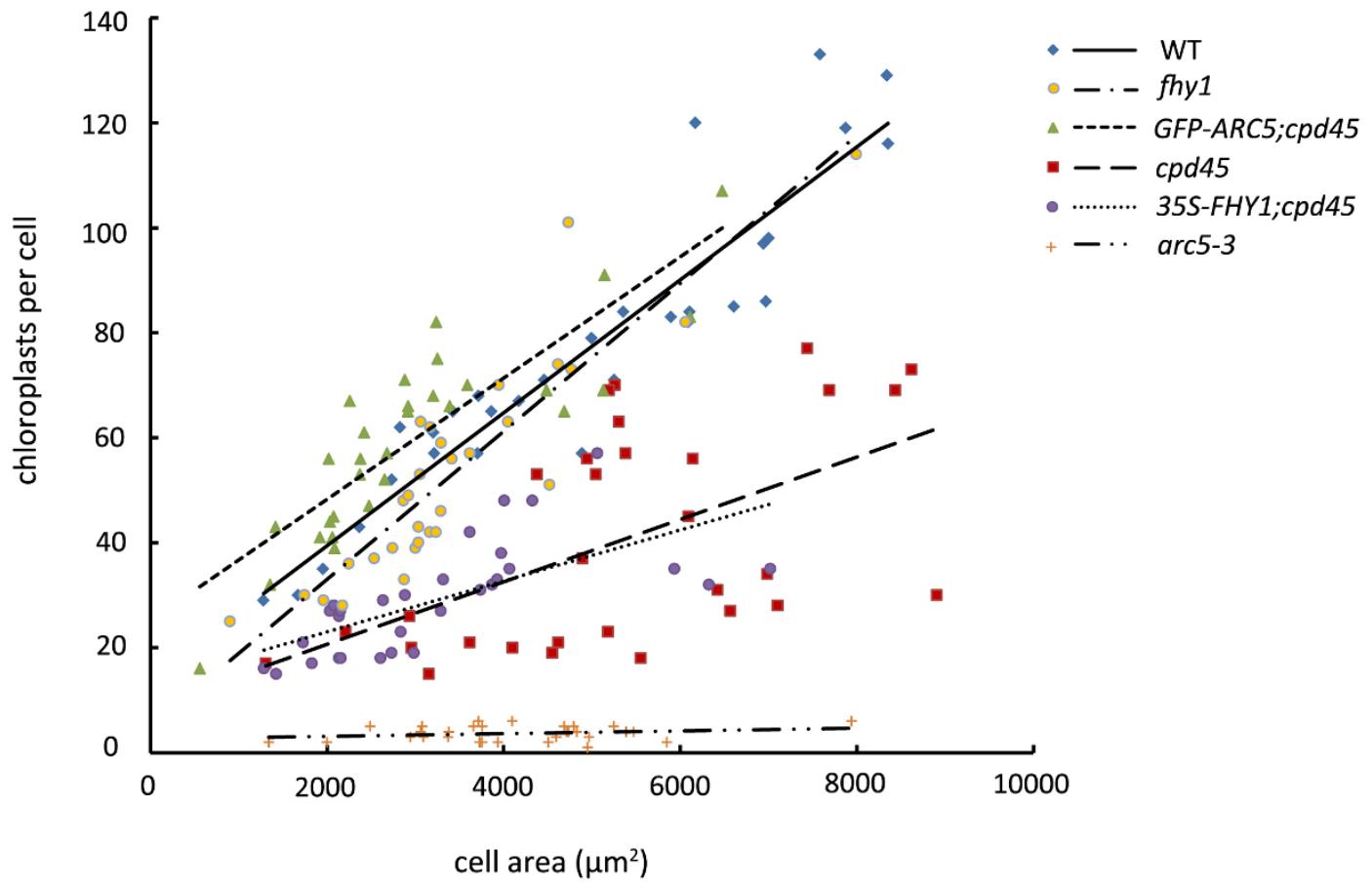

Figure 4 | Graph of chloroplast number relative to cell area of 40-day-old plants. The best-fit lines have slopes of $0.0127\left(\mathrm{R}^{2}=0.8701\right), 0.0141$ $\left(\mathrm{R}^{2}=0.8069\right), 0.0115\left(\mathrm{R}^{2}=0.7408\right), 0.0059\left(\mathrm{R}^{2}=0.2894\right), 0.0048\left(\mathrm{R}^{2}=0.4459\right)$, and $0.0003\left(\mathrm{R}^{2}=0.0554\right)$ for the wild type (WT), fhyl, GFPARC5;cpd45, cpd45, 35S-FHY1;cpd45, and arc5-3, respectively. 


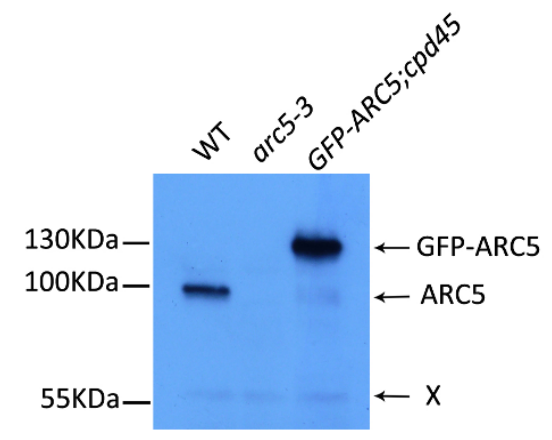

Figure $5 \mid$ Immunoblot analysis of ARC5 protein levels in wild-type, arc5-3, and GFP-ARC5; cpd45 plants. ARC5 and GFP-ARC5 are indicated, and were approximately $100 \mathrm{kD}$ and $130 \mathrm{kD}$ in size, respectively. X represents a non-specific band, which can be used as a loading control.

in the wild type, both in four-day-old seedlings grown under far-red light and in the leaves of 40-day-old plants grown under white light. Similarly, the expression level of ARC5 in $f h y 1$ was similar to that of the wild type under the above two conditions. The fhy 1 mutant used here (Salk_076131) has a T-DNA insertion in the first intron of FHY1 and disrupted gene expression (Figure S2). Thus, FHY1 and $A R C 5$ do not regulate each other's expression.

fhy1 is defective in far-red light signaling but has a normal chloroplast division phenotype. We measured the hypocotyl lengths of fhy 1 seedlings grown under far-red light or in the dark for 4 days and analyzed the chloroplasts of fhyl plants grown under white light for 40 days. Our results showed that the hypocotyl length of fhy 1 under far-red light was $8.432 \mathrm{~mm}$, a little more than three times that of the wild type $(2.772 \mathrm{~mm})$ (Figures 1a, e and 2). When grown in the dark, the hypocotyl length of fhyl was $12.72 \mathrm{~mm}$ (Figure 2a). fhyl had a chloroplast division phenotype similar to that of the wild type (Figure 3a, e). In the best-fit linear plot of chloroplasts per cell against cell area, the slope of fhy1 was 0.0141 , quite close to that of the wild type (0.0127; Figure 4$)$. This suggests

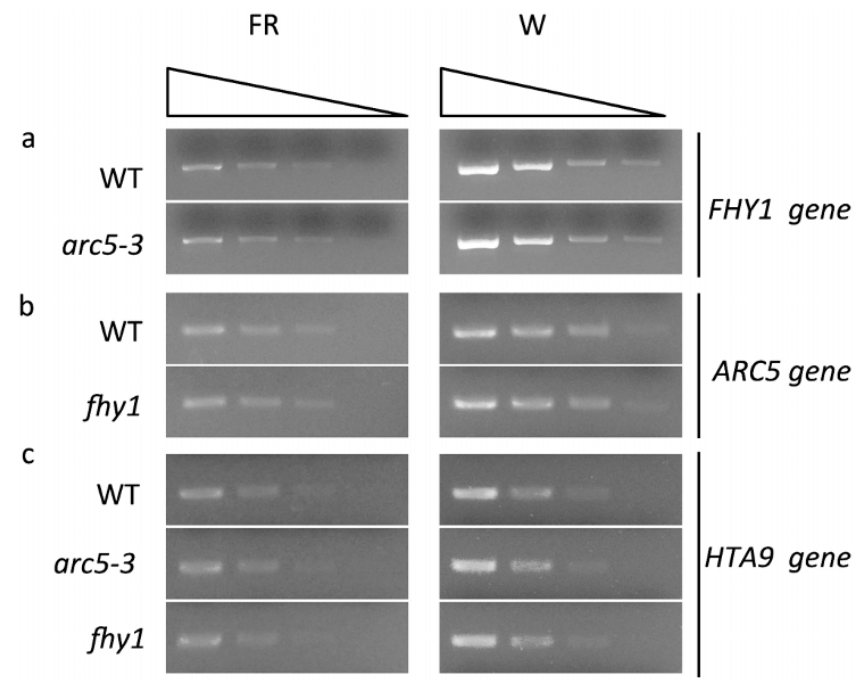

Figure 6 Semi-quantitative PCR analysis of gene expression level in wild type, arc5-3 and fhy1 plants. (A) FHY1 gene. (B) ARC5 gene. (C) HTA9 gene (HISTONE H2A PROTEIN 9). FR represents plants grown under farred light for 4 days and $\mathrm{W}$ represents plants grown under white light for 40 days. Black triangles indicate that the quantity of cDNA was serially diluted three times with a dilution factor of 4 (from left to right). HTA9 was used as a control. that FHY1 plays an important role in the far-red light signaling pathway, but not in chloroplast division or in the regulation of this process.

arc5-3 has an abnormal chloroplast division phenotype and a normal hypocotyl phenotype in far-red light. We measured the hypocotyl lengths and analyzed the chloroplast phenotype of the null allele, arc5-3, under the same growth conditions as above. arc5-3 exhibited a strikingly abnormal chloroplast phenotype, with very large and very few chloroplasts (Figure $3 \mathrm{f}$ ). The $\operatorname{arc5-3}$ mutant yielded a slope of 0.0003 in our best-fit linear plot of chloroplasts per cell against cell area (Figure 4), which was much smaller than that of cpd45 (0.0059) (Figure 4). By contrast, the hypocotyls of the arc5-3 mutants were of normal length $(2.817 \mathrm{~mm}$, versus $2.772 \mathrm{~mm}$ in the wild type; Figure 1a, f and Figure 2a). Thus, ARC5 is an important factor in the process of chloroplast division, but not in the far-red light signaling pathway.

\section{Discussion}

FHY3/CPD45 is required for the expression of both FHY1 and $A R C 5^{14,25}$. Previous studies showed that FHY1 is an important factor in the far-red light signaling pathway ${ }^{9,10,15,28}$. If FHY3/CPD45 regulated $A R C 5$ and chloroplast division through FHY1 and the far-red light signaling pathway, the chloroplast division defect of $c p d 45$ would be rescued when FHY1 expression was recovered. However, our results showed that the constitutive expression of FHY1 only rescued the far-red light signaling defect of $c p d 45$, but not the chloroplast division defect (Figures $1 \mathrm{c}$ and $3 \mathrm{c}$ ). This suggests that FHY1 does not function in the chloroplast division pathway. Furthermore, our study showed that fhy 1 had elongated hypocotyls under far-red light conditions, but that its mature leaves had a normal chloroplast division phenotype (Figures 1e and 3e), also suggesting that FHY1 does not function upstream of ARC5. Thus, FHY3/CPD45 regulates chloroplast division via a pathway that is distinct from far-red light signaling.

Furthermore, it was also unclear whether FHY3/CPD45 regulated far-red light signaling through the chloroplast division pathway or whether ARC5 and chloroplast division would affect far-red light signaling. The null allele of $A R C 5, \operatorname{arc5}-3$, exhibited a very severe chloroplast division phenotype (Figures $3 \mathrm{f}$ and 4 ), but its response to far-red light was similar to that of the wild type (Figures $1 \mathrm{f}$ and 2). This indicates that ARC5 is an important factor in chloroplast division, but not in the far-red light signaling pathway. Moreover, a transgene of GFP-ARC5 driven by the $35 \mathrm{~S}$ promoter only rescued the chloroplast division defect of $c p d 45$ (Figure $3 \mathrm{~d}$ ), but not the farred light signaling defect (Figure 1d). These results suggest that ARC5 does not function upstream of FHY1.

FHY1 has been shown to be required for the nuclear import of phyA and thus the far-red light signaling ${ }^{29}$. As shown in Figures 1 and 2, the fhy 1 mutant had a defect of far-red light sensing like $c p d 45$. However, fhy 1 seemed to be not completely blind to far-red light like cpd45, because the elongation of its hypocotyls was slightly inhibited by far-red light (Figure 2). FHL had been shown to have some redundancy with FHY1 for the nuclear import of phyA, but with a minor role ${ }^{10,12}$. So, a small part of phyA could be imported into the nucleus with the assistance of FHL for far-red light sensing. Alternatively but unlikely, the T-DNA in fhyl was inserted into an intron of FHY1 gene (Figure S2), so that there might be still a very small amount of correctly spliced mRNA.

Seedlings of 35S-FHY1;cpd45 appeared to be stronger than the others both in far-red light or the dark (Figure 1 and S1). Especially, the cotyledon size appeared to be larger. 35S promoter is a constitutive and very strong promoter. It could cause an overexpression of FHY1. This might increase the nuclear import efficiency of phyA or other proteins and affect the development of plants. 
In contrast to the complete absence of ARC5 in arc5-3, our immunoblot analysis suggested that there was still a low level of ARC5 in cpd45 (Figure 5). This is probably because the EMS-induced point mutation in cpd45 only caused an amino acid substitution in the encoded protein, resulting in a leaky mutation ${ }^{25}$. Therefore, there is still some trace activity of FHY3/CPD45 and a very slight activation of $A R C 5$ expression in $c p d 45$. Alternatively, $c p d 45$ may have totally lost its activity and the ARC5 expression may be due only to basal expression. The lack of ARC5 in arc5-3 could be due to the incorrect splicing of the pre-mRNA, which introduced a premature stop $\operatorname{codon}^{25}$. Regardless, the low level, but not the absence, of ARC5 in cpd45 can explain why its chloroplast division defect is not as severe as that of $\operatorname{arc5}-3$.

Our results also suggest that the constitutive overexpression of GFP-ARC5 can rescue the chloroplast division defect in cpd45 (Figure 3d). The level GFP-ARC5 in the overexpressing plants is at least five times greater than the level of ARC5 in the wild type (Figure 5). However, previous studies have shown that overexpression of a chloroplast division protein, such as FtsZ, MinD, or MinE, could inhibit chloroplast division ${ }^{30-32}$. This is probably because these proteins form a complex with a stringent stoichiometry and overexpression of one component causes misassembly of the complex and then faulty chloroplast division. The working mechanism of ARC5 may be different from that of other chloroplast division proteins.

Our gene expression analysis showed that expression of FHY1 or $A R C 5$ was not substantially affected in the alternative mutant under both white light and far-red light conditions (Figure 6). This further suggests that these two genes do not function in the same pathway, i.e., that they do not function upstream or downstream of each other. Instead, they are both regulated by FHY3/CPD45 in parallel.

Many lines of evidence suggest that FHY3/CPD45 regulates circadian rhythm in Arabidopsis ${ }^{33-35}$. For instance, FHY3/CPD45 regulates the expression of ELF4 (Early Flower 4) and plays a dominant role in the CCA1/LHY-TOC1 circadian clock feedback circuit ${ }^{36,37}$, by binding to the ELF4 promoter, and thereby regulates the biological clock of plants ${ }^{35}$.

Collectively, the information presented in this and previous studies suggest that FHY3/CPD45 is a crunode in the gene regulatory network, which links pathways of photomorphogenesis, circadian rhythm, chloroplast division and others. Therefore, we propose a genetic model of $F H Y 3 / C P D 45$-mediated gene regulatory pathways (Figure 7). In this model, FHY3/CPD45 is an important regulator of many genes that controls chloroplast division, far-red light signaling, and the circadian rhythm through regulating the expression of $A R C 5, F H Y 1$, and ELF4, respectively. As an important transcription factor, FHY3/CPD45 may also regulate the expression of many other genes and govern many other biological pathways.

This work has finally clarified that the relationship between FHY1 and $A R C 5$ in the gene regulatory network is parallel. It is well known that light signals are essential regulators of circadian rhythm and many other biological processes. Therefore, future studies should investigate the relationship between the far-red light signaling pathway and circadian rhythm and other pathways. Such studies may reveal a complex gene regulatory network mediated by FHY3/ CPD45.

\section{Methods}

Plant materials. All Arabidopsis thaliana plants used in this study were in the Columbia-0 ecotype background. $\operatorname{arc5-3}$ is a mutant allele of $A R C 5$, and $c p d 45$ is a mutant allele of $F H Y 3^{25}$. fhy1 (Salk_076131) is a T-DNA insertion mutant of FHY1.

Growth conditions. Sterilized seeds were plated on 1/2 MS medium with $1 \%$ sucrose and $0.8 \%$ agar. After stratification at $4{ }^{\circ} \mathrm{C}$ for 2 days, the plates were placed vertically under continuous far-red light $\left(720 \sim 740 \mathrm{~nm}, 10 \mu \mathrm{mol} \cdot \mathrm{m}^{-2} \cdot \mathrm{s}^{-1}\right)$ or in the dark for 4 days without photoperiod at $22^{\circ} \mathrm{C}$ as before Ref. 25 . Soil-grown plants were grown from seed in a growth chamber under white light $\left(90 \sim 120 \mu \mathrm{mol} \cdot \mathrm{m}^{-2} \cdot \mathrm{s}^{-1}\right)$ with 40 $\sim 60 \%$ relative humidity at $22^{\circ} \mathrm{C}$ and a photoperiod of 16 -h-light/8-h-dark.

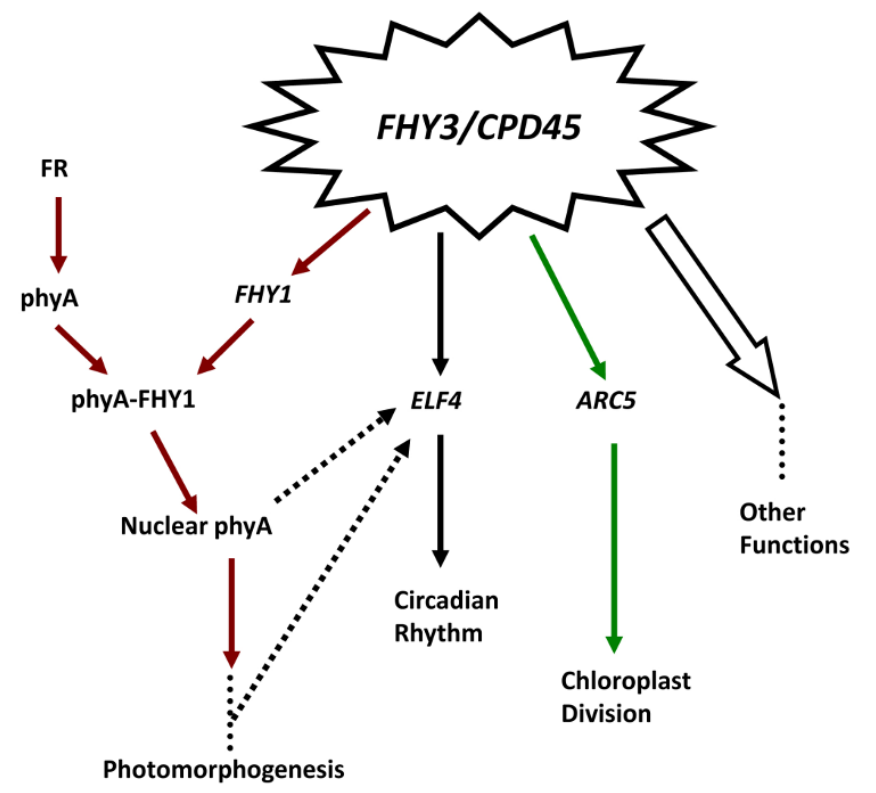

Figure 7 | A simplified genetic model of $F H Y 3 / C P D 45$-mediated gene regulatory pathways. CPD45 (also known as FHY3) regulates the expression of many genes and is an important regulator of chloroplast division, the far red-light signaling pathway, circadian rhythm, and other biological processes. Arrows indicate genes that are directly regulated by FHY3/CPD45 and the pathways in which they are involved, dotted arrows indicate possible pathways that remain to be confirmed, and dotted lines represent unknown components or factors and steps not indicated.

Measurement of hypocotyl length and cotyledon size. Plates were removed from the far-red light or dark box after 4 days of growth. Images were first taken with a dissection microscope and a USB2.0 digital camera (Changheng, Beijing) and then the hypocotyl length and cotyledon size (area) were measured with the software Image Analysis System 10.0 (Changheng, http://www.crisoptical.com/lm2_41_344. $\mathrm{htm})$. The sample size was 30 .

Leaf fixation and chloroplast phenotype analysis. To characterize the chloroplast phenotype, leaf tissues from 40-day-old plants were fixed in an Eppendorf tube with $3.5 \%$ glutaraldehyde for $1 \mathrm{~h}$ in darkness, the glutaraldehyde was then replaced with $0.1 \mathrm{M} \mathrm{Na}_{2} \mathrm{EDTA}(\mathrm{PH}=9.0)$, and the tube was placed in a water bath of $55^{\circ} \mathrm{C}$ for $2 \mathrm{~h}$. Images were taken with a USB2.0 digital camera (Changheng, Beijing) coupled to an Olympus CX21 microscope (Olympus, Tokyo). The chloroplast division phenotype was quantified as described previously ${ }^{25}$. Thirty mesophyll cells of each group were used for the quantification.

Identification of an fhy1 T-DNA insertion mutant, Salk_076131. The fhyl T-DNA insertion mutant Salk_076131 was obtained from the Arabidopsis Biological Resource Center. The sequence flanking the T-DNA insertion site was amplified using primers LBal (5'- TGG TTC ACG TAG TGG GCC ATC G -3') and RP (5'TGG TAG GCT TCT TTG TCT CAT G -3') and sequenced. The T-DNA insertion site was then verified by sequence analysis.

Plasmid construction. FHY1 were amplified from the genomic DNA of wild-type plants using primers 5'- GCG ATC TAT GCC TGA AGT GG -3' and 5'- CGA CCA TGG GAT ACT CTT GAA CAC AAG ATT GG -3' and then inserted into $3302 \mathrm{Y} 3$ between the StuI and NcoI cutting sites to generate 35S-FHY1. 35S-GFP-ARC5 was constructed in a previous study ${ }^{25}$. To obtain the pET30a-ARC5 expression vector, $A R C 5$ was amplified from the wild-type cDNA with the primers 5'- GGA GGA TCC GAG CGG TGG AGT CTT TAC G - 3' and 5'- CCA CTC GAG AAA GCT CAG GAT CAA CTT GCA TCA C - 3 '. The PCR product was cloned into the pET30a vector between the XhoI and XbaI sites.

RNA extraction and semi-quantitative RT-PCR analysis. RNA was isolated from seedlings grown on plates under far-red light for 4 days or from the leaves of 40-dayold plants grown in soil under white light, using an RNApure Total RNA Isolation Kit (Aidlab, Beijing) and reverse transcribed with a RevertAid First Strand cDNA Synthesis Kit (Thermo Fisher). Semi-quantitative RT-PCR analysis was performed as before Ref. 38. The cDNA templates used for PCR were serially diluted three times at a dilution ratio of 4. FHY1 was amplified with the primers 5'- GCG ATC TAT GCC TGA AGT GG -3' and 5'- GAG TAG AAT CAT CTT GGT TAA CAG TCC - 3 '. ARC5 was amplified with the primers 5'- GCT AAT ACC GAA TGC AGG GAT G -3' and 5'- GCC TTC GCA ACT GCT ATA ACA C -3'. 
Protein purification, antibody preparation and immuno-blot analysis. The pET30a-ARC5 plasmid was isolated and transformed into the Rosetta strain of E. coli for protein expression. Bacterial cultures were incubated at $37^{\circ} \mathrm{C}$ until the $\mathrm{OD}_{600}$ reached $0.4-0.6$. Then, $1 \mathrm{mM}$ IPTG(isopropyl $\beta$-D-thiogalactopyranoside) was added to the culture to induce the expression of ARC5. Bacterial cells were lysed by ultra-sonication and the induced ARC5 protein was purified using Ni Sepharose 6 Fast Flow (GE Healthcare). The polyclonal and monoclonal antibodies against ARC5 were raised in mouse. For immuno-blot analysis, proteins were separated by SDSPAGE and transferred to Immun-Blot PVDF membrane (Bio-Rad). Blots were probed with anti-ARC5 mouse monoclonal antibodies at a dilution of $1: 1000$ for $1 \mathrm{~h}$. Secondary antibodies of the Goat anti-Mouse IgG-conjugated HRP (1:10000 dilution) and an eECL Western Blot Kit (Beijing ComWin Biotech Company) were then used.

1. Sharrock, R. A. \& Quail, P. H. Novel phytochrome sequences in Arabidopsis thaliana: structure, evolution, and differential expression of a plant regulatory photoreceptor family. Genes Dev 3, 1745-57 (1989).

2. Chaves, I. et al. The Cryptochromes: Blue Light Photoreceptors in Plants and Animals. Annual Review of Plant Biology, Vol 62 62, 335-364 (2011).

3. Briggs, W. R. \& Christie, J. M. Phototropins 1 and 2: versatile plant blue-light receptors. Trends Plant Sci 7, 204-10 (2002).

4. Kim, W. Y. et al. ZEITLUPE is a circadian photoreceptor stabilized by GIGANTEA in blue light. Nature 449, 356-60 (2007)

5. Heijde, M. \& Ulm, R. UV-B photoreceptor-mediated signalling in plants. Trends in Plant Science 17, 230-237 (2012).

6. Rizzini, L. et al. Perception of UV-B by the Arabidopsis UVR8 protein. Science 332, 103-6 (2011).

7. Quail, P. H. The phytochrome family: dissection of functional roles and signalling pathways among family members. Philos Trans R Soc Lond B Biol Sci 353, 1399-403 (1998)

8. Nagy, F. \& Schafer, E. Phytochromes control photomorphogenesis by differentially regulated, interacting signaling pathways in higher plants. Annu Rev Plant Biol 53, 329-55 (2002).

9. Hiltbrunner, A. et al. Nuclear accumulation of the phytochrome A photoreceptor requires FHY1. Current Biology 15, 2125-30 (2005).

10. Rausenberger, J. et al. Photoconversion and nuclear trafficking cycles determine phytochrome A's response profile to far-red light. Cell 146, 813-25 (2011).

11. Pfeiffer, A. et al. A cell-free system for light-dependent nuclear import of phytochrome. Plant Journal 57, 680-689 (2009).

12. Zhou, Q. Q. et al. FHL is required for full phytochrome A signaling and shares overlapping functions with FHY1. Plant Journal 43, 356-370 (2005)

13. Hudson, M., Ringli, C., Boylan, M. T. \& Quail, P. H. The FAR1 locus encodes a novel nuclear protein specific to phytochrome A signaling. Genes Dev 13, 2017-27 (1999).

14. Lin, R. C. et al. Transposase-derived transcription factors regulate light signaling in Arabidopsis. Science 318, 1302-1305 (2007).

15. Desnos, T., Puente, P., Whitelam, G. C. \& Harberd, N. P. FHY1: a phytochrome Aspecific signal transducer. Genes Dev 15, 2980-90 (2001).

16. Wang, H. \& Deng, X. W. Arabidopsis FHY3 defines a key phytochrome A signaling component directly interacting with its homologous partner FAR1. EMBO J 21, 1339-49 (2002).

17. Dehesh, K. et al. Arabidopsis HY8 locus encodes phytochrome A. Plant Cell 5, 1081-8 (1993).

18. Bates, P. D. et al. Fatty acid synthesis is inhibited by inefficient utilization of unusual fatty acids for glycerolipid assembly. Proc Natl Acad Sci U S A 111, 1204-9 (2014)

19. Noctor, G., Arisi, A. C., Jouanin, L. \& Foyer, C. H. Manipulation of glutathione and amino acid biosynthesis in the chloroplast. Plant Physiol 118, 471-82 (1998).

20. Seay, M., Hayward, A. P., Tsao, J. \& Dinesh-Kumar, S. P. Something old something new: plant innate immunity and autophagy. Curr Top Microbiol Immunol 335, 287-306 (2009).

21. Osteryoung, K. W. \& Nunnari, J. The division of endosymbiotic organelles. Science 302, 1698-704 (2003).

22. Koniger, M., Delamaide, J. A., Marlow, E. D. \& Harris, G. C. Arabidopsis thaliana leaves with altered chloroplast numbers and chloroplast movement exhibit impaired adjustments to both low and high light. Journal of Experimental Botany 59, 2285-2297 (2008).

23. Jeong, W. J. et al. A large population of small chloroplasts in tobacco leaf cells allows more effective chloroplast movement than a few enlarged chloroplasts. Plant Physiol 129, 112-21 (2002).
24. Gao, H. B., Kadirjan-Kalbach, D., Froehlich, J. E. \& Osteryoung, K. W. ARC5, a cytosolic dynamin-like protein from plants, is part of the chloroplast division machinery. Proceedings of the National Academy of Sciences of the United States of America 100, 4328-4333 (2003).

25. Gao, Y. F. et al. Arabidopsis FRS4/CPD25 and FHY3/CPD45 work cooperatively to promote the expression of the chloroplast division gene ARC5 and chloroplast division. Plant Journal 75, 795-807 (2013).

26. Lepisto, A. \& Rintamaki, E. Coordination of Plastid and Light Signaling Pathways upon Development of Arabidopsis Leaves under Various Photoperiods. Molecular Plant 5, 799-816 (2012).

27. Ouyang, X. H. et al. Genome-Wide Binding Site Analysis of FAR-RED ELONGATED HYPOCOTYL3 Reveals Its Novel Function in Arabidopsis Development. Plant Cell 23, 2514-2535 (2011).

28. Genoud, T. et al. FHY1 Mediates Nuclear Import of the Light-Activated Phytochrome A Photoreceptor. Plos Genetics 4, e1000143 (2008).

29. Hiltbrunner, A. et al. Nuclear accumulation of the phytochrome A photoreceptor requires FHY1. Current Biology 15, 2125-2130 (2005).

30. Stokes, K. D., McAndrew, R. S., Figueroa, R., Vitha, S. \& Osteryoung, K. W. Chloroplast division and morphology are differentially affected by overexpression of FtsZ1 and FtsZ2 genes in Arabidopsis. Plant Physiol 124, 1668-77 (2000).

31. Dinkins, R., Reddy, M. S., Leng, M. \& Collins, G. B. Overexpression of the Arabidopsis thaliana MinD1 gene alters chloroplast size and number in transgenic tobacco plants. Planta 214, 180-8 (2001).

32. Reddy, M. S., Dinkins, R. \& Collins, G. B. Overexpression of the Arabidopsis thaliana MinE1 bacterial division inhibitor homologue gene alters chloroplast size and morphology in transgenic Arabidopsis and tobacco plants. Planta 215 , $167-76$ (2002).

33. Yanovsky, M. J., Mazzella, M. A., Whitelam, G. C. \& Casal, J. J. Resetting of the circadian clock by phytochromes and cryptochromes in Arabidopsis. J Biol Rhythms 16, 523-30 (2001).

34. Allen, T. et al. Arabidopsis FHY3 specifically gates phytochrome signaling to the circadian clock. Plant Cell 18, 2506-16 (2006)

35. Li, G. et al. Coordinated transcriptional regulation underlying the circadian clock in Arabidopsis. Nat Cell Biol 13, 616-22 (2011).

36. Kikis, E. A., Khanna, R. \& Quail, P. H. ELF4 is a phytochrome-regulated component of a negativefeedback loop involving the central oscillator components CCA1 and LHY. Plant Journal 44, 300-313 (2005).

37. McWatters, H. G. et al. ELF4 is required for oscillatory properties of the circadian clock. Plant Physiology 144, 391-401 (2007).

38. Pan, D. et al. Genetic mapping and isolation of two arc3 alleles in Arabidopsis. Plant Cell Rep 32, 173-82 (2013).

\section{Acknowledgments}

We would like to thank the SALK institute and ABRC for providing the Salk_076131 T-DNA line. This work was supported by the National Natural Science Foundation of China (30971439, J1103516), the 111 Project (B13007) and the Fundamental Research Funds for the Central Universities.

\section{Author contributions}

N.C., Y.G. and L.Z. performed the experiments. N.C., Y.G. and X.L. prepared the manuscript. H.G. provided advice on the experiments and improved the manuscript.

\section{Additional information}

Supplementary information accompanies this paper at http://www.nature.com/ scientificreports

Competing financial interests: The authors declare no competing financial interests. How to cite this article: Chang, N., Gao, Y., Zhao, L., Liu, X. \& Gao, H. Arabidopsis FHY3/ CPD45 regulates far-red light signaling and chloroplast division in parallel. Sci. Rep. 5, 9612; DOI:10.1038/srep09612 (2015)

This work is licensed under a Creative Commons Attribution 4.0 International License. The images or other third party material in this article are included in the article's Creative Commons license, unless indicated otherwise in the credit line; if the material is not included under the Creative Commons license, users will need to obtain permission from the license holder in order to reproduce the material. To view a copy of this license, visit http://creativecommons.org/licenses/by/4.0/ 